\title{
Automated feedback controlled charging circuitry for lithium ion battery
}

\author{
Dheerendra Singh ${ }^{1}$, Shivam Khandelwal ${ }^{2}$ and Parakh Sarda ${ }^{2 *}$ \\ Assistant Professor, Birla Institute of Technology and Science, Pilani, India ${ }^{1}$ \\ Undergraduate Student, Birla Institute of Technology and Science, Pilani, India ${ }^{2}$ \\ (C2016 ACCENTS
}

\begin{abstract}
The number of mobile phone devices in India, a country having a population of about 1.252 billion is about one billion, and rising gradually, owing to rising economy and cheap mobile phones coming up each day. In such situations, the importance of developing efficient charging systems becomes exponentially high. This paper attempts to propose the model for an efficient automated microcontroller controlled battery charging system, that takes in the feedback from the device, by detecting the state of charge (SOC) of the battery, and then algorithmically detects the charging phase in which the charger has to charge the lithium ion battery. The model proposed is developed in MATLAB/Simulink, and an iterative procedure is followed to determine the closest voltage values for most efficient charging. Once these characteristics of batteries are detected, the statistics gathered will be used to model the circuit for fast charging of the battery using the algorithm developed.
\end{abstract}

\section{Keywords}

Battery, Charging, Feedback, Automation, Design proposal, Simulation, MALTAB/Simulink.

\section{Introduction}

The paper focuses on automation of the control process of lithium ion battery characteristic determination cycle and developing an automated charger based on feedback from the battery. There is an ever growing demand for Li-Ion batteries in consumer market as Li-Ion batteries can store more energy than that of Ni-Cd batteries with the same size and weight. Due to their small size, light weight and the rechargeable characteristics, Li-Ion battery is well suited to portable electronic applications. Also, unlike many other types of rechargeable batteries, Lithium-Ion batteries do not need priming. The first charge of a Li-Ion battery is no different than the 10 th or the 100th charge. Lithium-ion batteries may be - and should be charged often. The battery lasts longer with partial rather than full discharges. Full discharges should be avoided because of wear. The battery loses capacity due to aging, whether used or not. The paper starts with a theoretical review of the ideal charging profile of li-ion battery in section 2 , followed by section 3 , which proposes a model for automated circuit that will be used to make an efficient, fast charger based on voltage feedback from the battery terminals.

\footnotetext{
*Author for correspondence
}

Section 4 highlights the developed MATLAB/Simulink model and the results obtained by iterative simulations of the model with different combinations of constant current and constant voltage values, and comparing the results obtained to determine the most appropriate values for charging.

\section{Ideal lithium ion battery charging profile}

The li-ion battery, unlike any other types of battery requires a specific kind of charging input for efficient charging. There are three phases of charging a li-ion battery, viz. constant current phase, followed by the constant voltage phase, and then the additional topping charge phase. The CV charging method is easy to control and safe, but it requires a longer time to charge. The CC charging method mainly charges a battery by a constant current. The advantage is the decrement of charging time when a larger charging current is used. Yet, by continuously charging the battery with a larger current without realizing the maximum charge limit, it may result in overcharging and cause the unexpected damage to the battery. Choosing a lower voltage threshold, or eliminating the saturation charge altogether, prolongs battery life but this reduces the runtime. The theoretical study of the three phases is specified as below: 
Constant current phase: A constant current, typically closer to $1 \mathrm{C}$ is applied to make the battery reach a level of 70-80\% charge by varying the voltage that is applied [1].

Constant voltage phase: After reaching $70-80 \%$ of the capacity, the battery is charged at a constant voltage, determined by the battery characteristics. The battery reaches a charged capacity of about 90 $100 \%$ after this stage, after which the charger is disconnected.

Additional topping charge phase: In this phase, after turning off the charger, when the terminal potential starts to fall, the additional current is provided [2].

Figure 1 represents the three phases graphically.

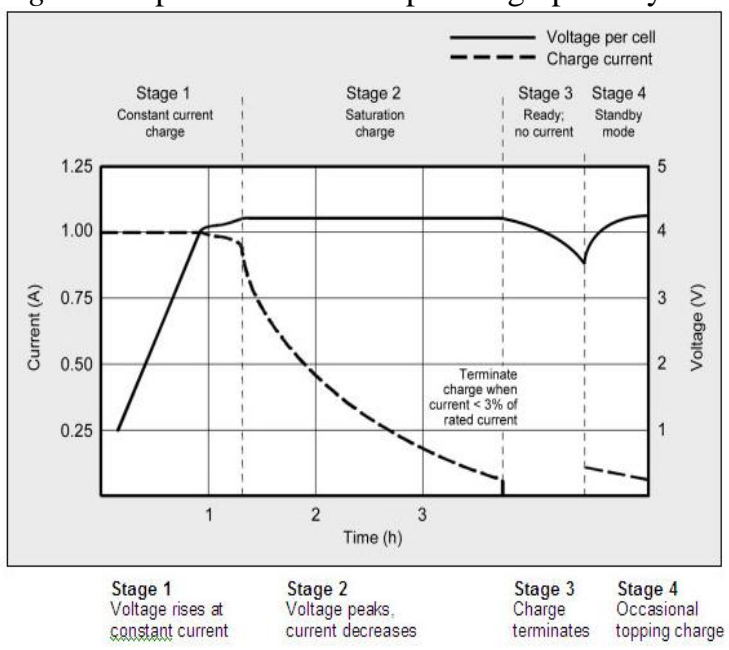

Figure 1 Charging profile of a Li-Ion battery (Courtesy of www.batteryuniversity.com)

\section{Manual procedure}

3.1Manually determined charging cycle profile of Li-ion battery

Experimental setup consisting of buck converter, DC source, microcontroller and battery was assembled to determine the charging profile of a $4.2 \mathrm{~V} / 3.7 \mathrm{~V}(1650 \mathrm{mAh})$ Li-Ion battery manually[3, 4], by taking the rested terminal voltage of the battery at regular intervals during its charging. The charging profile is used as a reference to find out the Constant Current and Constant Voltage phases of the battery which will be used in the proposed model. The characteristics of buck converter used, were determined by the procedure given in section 3.2, to be used as reference in the fast charging model circuit [5].
A sample code for microcontroller open source platform was developed, in which a Pulse Width Modulated (PWM) output was generated by microcontroller with appropriate duty cycle depending on the battery's terminal potential and circuit current and the PWM output of the microcontroller was applied to the buck converter gate input. The following assumptions were made in the micro-controller code while testing the battery for charging.

- Battery dead or degraded if the cell voltage is below $3.3 \mathrm{~V}$.

- Constant current phase when the battery voltage is between 3.3 and $4.02 \mathrm{~V}$ wherein the output voltage from the buck converter is $7-9 \mathrm{~V}$ varying linearly. The charging current is decided by the terminal voltage of the batteries subtracted from the charging voltage, and then divided by the internal resistance of the batteries.

- Constant voltage phase when the battery voltage is between 4.02 and $4.2 \mathrm{~V}$ wherein the output is a constant $5 \mathrm{~V}$.

- Battery fully charged if the battery terminal voltage is $4.2 \mathrm{~V}$ or above. The output is then cut off.

- A resistive load rated at $2 \Omega / 5 \mathrm{~W}$ was used in the initial setup in series with the battery to monitor the current in the circuit for protecting the battery from excessive current in the circuit [6].

\subsection{Proteus model for the experimental setup}

The charging circuitry uses an AC source which is stepped down by a step down transformer to an output voltage of $18 \mathrm{~V}$. The stepped down output is rectified using a full wave rectifier. This output is controlled by switching MOSFETs driven by the microcontroller to obtain the desired voltage for charging. The circuit uses an AVR based CISC micro-controller for controlling various parameters of the circuit, however, any other microcontroller may be used for serving the same purpose. A digital potentiometer is used to act as a variable resistance controlled by the micro-controller which is used for current control of the circuit. The circuit is disconnected at regular intervals of 1 minute by using MOSFETs as control switch and feedback is taken from the battery terminals to control the charging process. The MOSFETs switch alternatively as opposite signals are applied on the gate with the help of a NOT gate. The Proteus model in Figure 2 shows the various components used in the circuit [7]. 
The proposed model was tested by controlling the charging of the Li-Ion battery by microcontroller and charging the battery by controlling the DC voltage output of a Buck Converter. The current in the circuit was controlled by varying the digital potentiometer's resistance and thus gaining the current control of the circuitry [8].
The terminal potential across the battery and the charging current was observed at regular intervals. Figure 3 shows the rested terminal voltage across the battery with respect to time and Figure 8 shows the charging current.

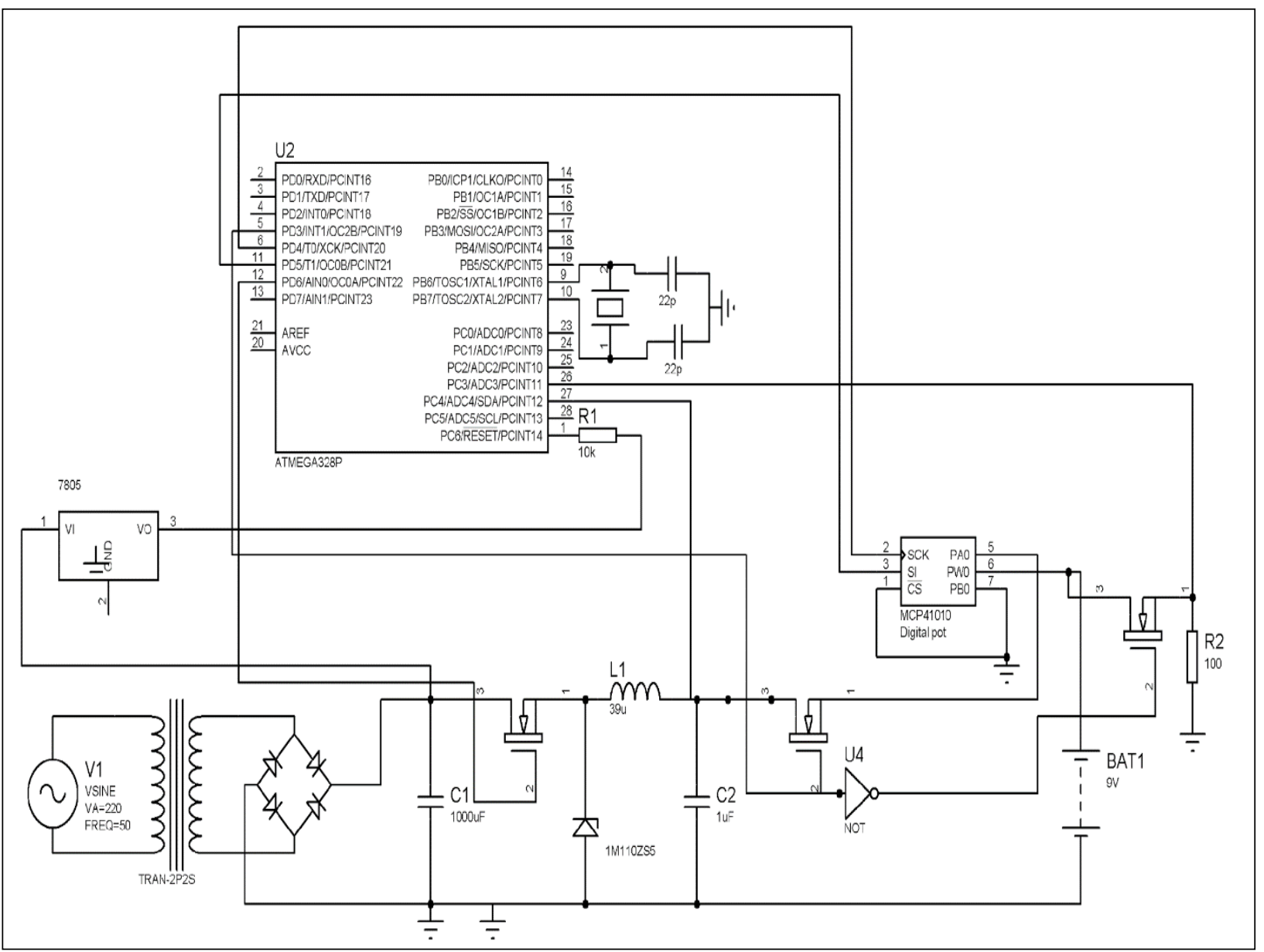

Figure 2 Proteus model

\subsection{Results obtained}

To make sure that the circuit proposed is realizable and will lead to appreciable results, a single physical implementation was carried out in the lab. The manual implementation of this circuitry for fast charging decreased the charging time from 133 minutes, determined previously, to about 73 minutes, for 0 to $95 \%$ charging. The following graphs shows the battery voltage plotted against time (Figure 3) and charging current plotted against time (Figure 4).

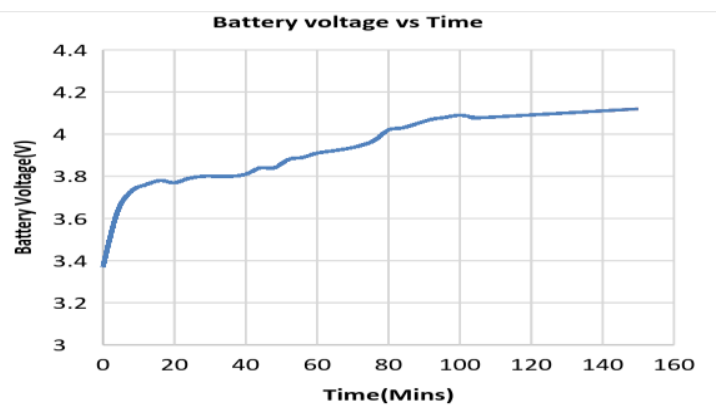

Figure 3 Battery terminal potential vs. time for proposed charger 


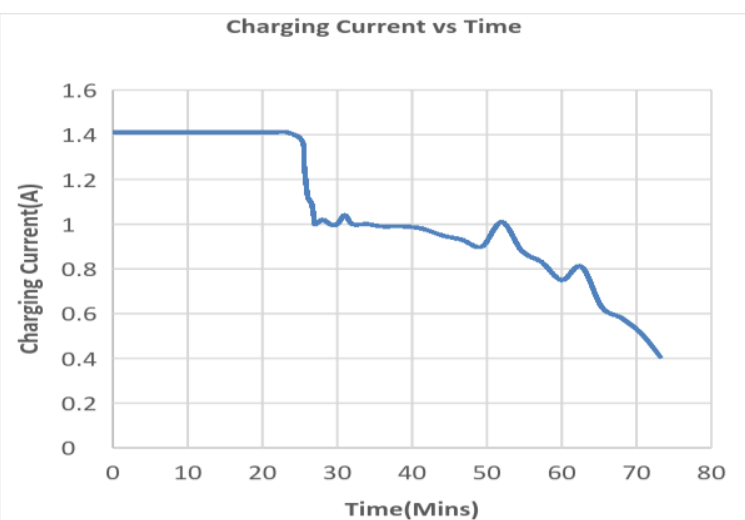

Figure 4 Current vs. time curve for proposed charger

\section{MATLAB/ Simulink simulation for charger}

A MATLAB/Simulink model was developed to simulate the proposed circuitry with varying current and voltage values, thus enabling analysis and further design improvements for the same. A pre-installed model of battery provided in MATLAB rated at 3.6V/2050mAh Li-Ion battery was used for the same. Due to the limitations of circuit elements availability and inter connectivity of elements of different libraries in MATLAB, and the simulation time required for operations involving too many calculations, two different circuits were implemented. The first circuits generated accurate charging voltage based on requirement of constant current or constant voltage, by taking feedback from the battery itself. This circuit was slower for simulation purposes, hence generating a need to use faster, less computational circuit to plot the entire charging and discharging patterns $[9,10]$.

Figure 5 and Figure 6 display the circuits developed in MATLAB.

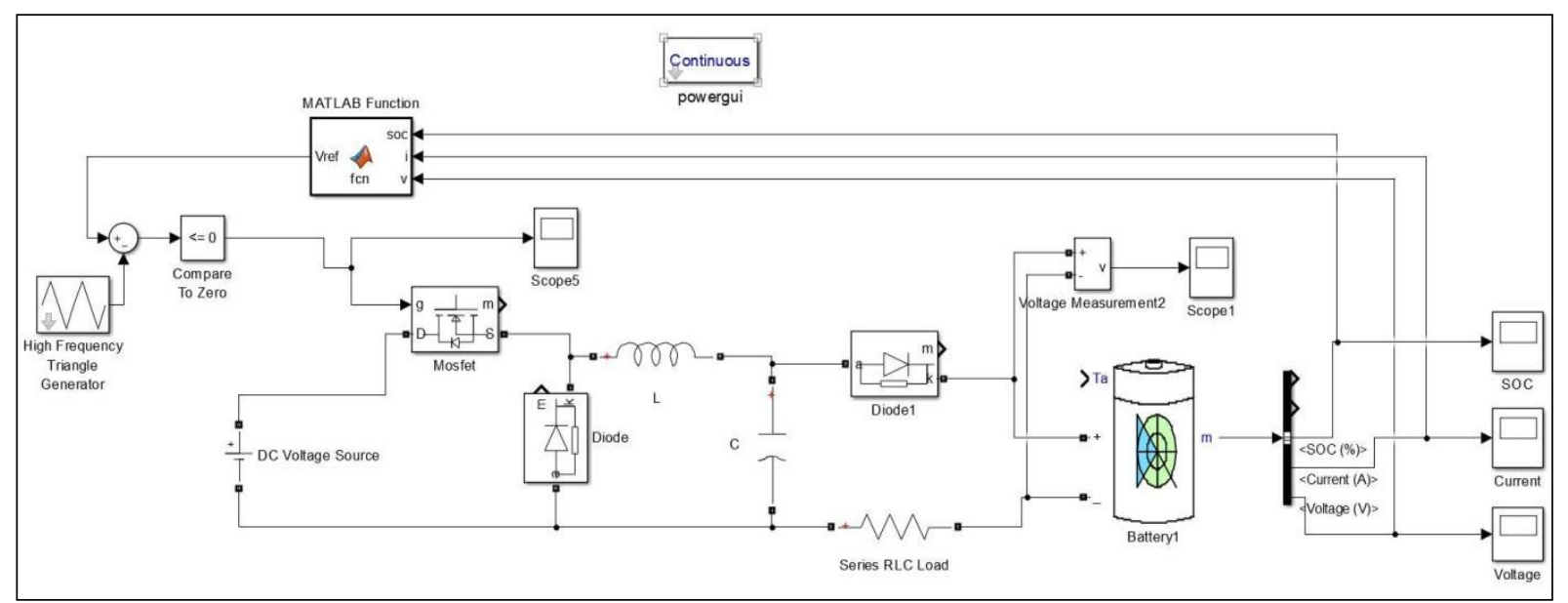

Figure 5 Feedback controlled charging circuitry for charging Li-Ion battery model in simulink

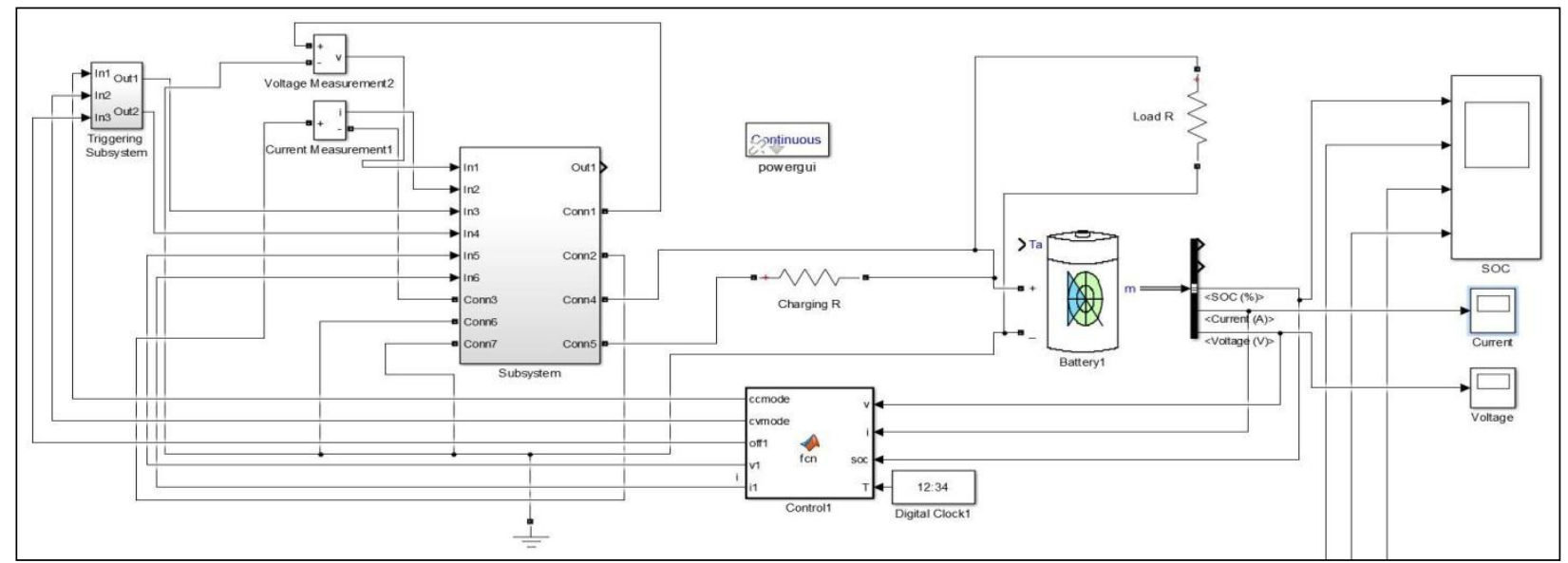

Figure 6 Fast simulation circuitry involving feedback from battery and controlled charging model in simulink 
Dheerendra Singh et al.

\subsection{Simulation results}

After running the simulation for 7000 seconds, and iteratively varying the values of the constant current and the constant voltage that is used for charging. The preliminary results obtained are plotted in Figure
8. The simulation results show significant improvement against the normal battery charger, whose charging profiles are plotted in Figure 7, reducing the charging time by almost $25-30 \%$.

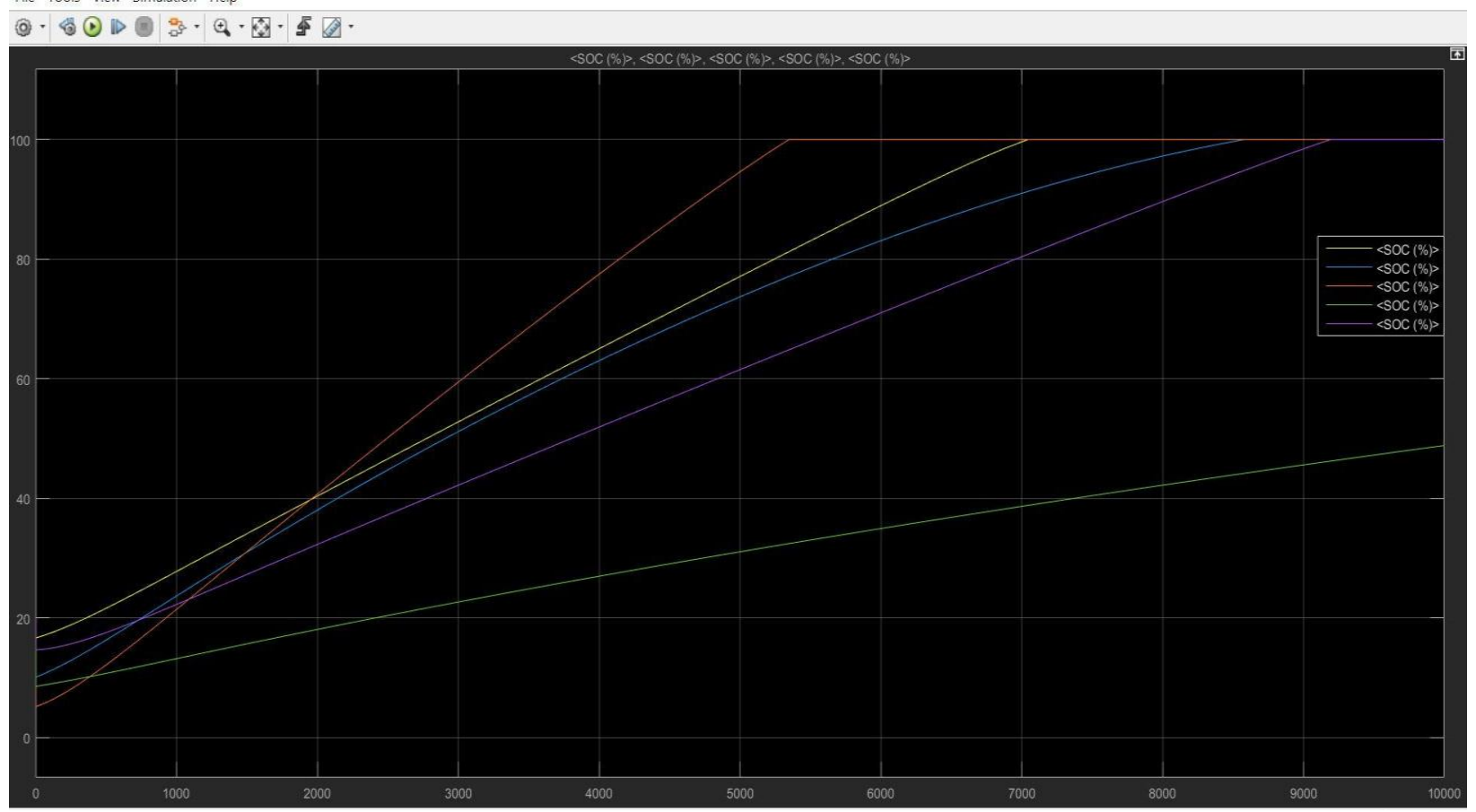

Figure 7 Charging profile of normal battery charger (Battery SOC)

$$
\text { A SOC }
$$

File Tools View Simulation Help

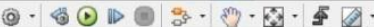

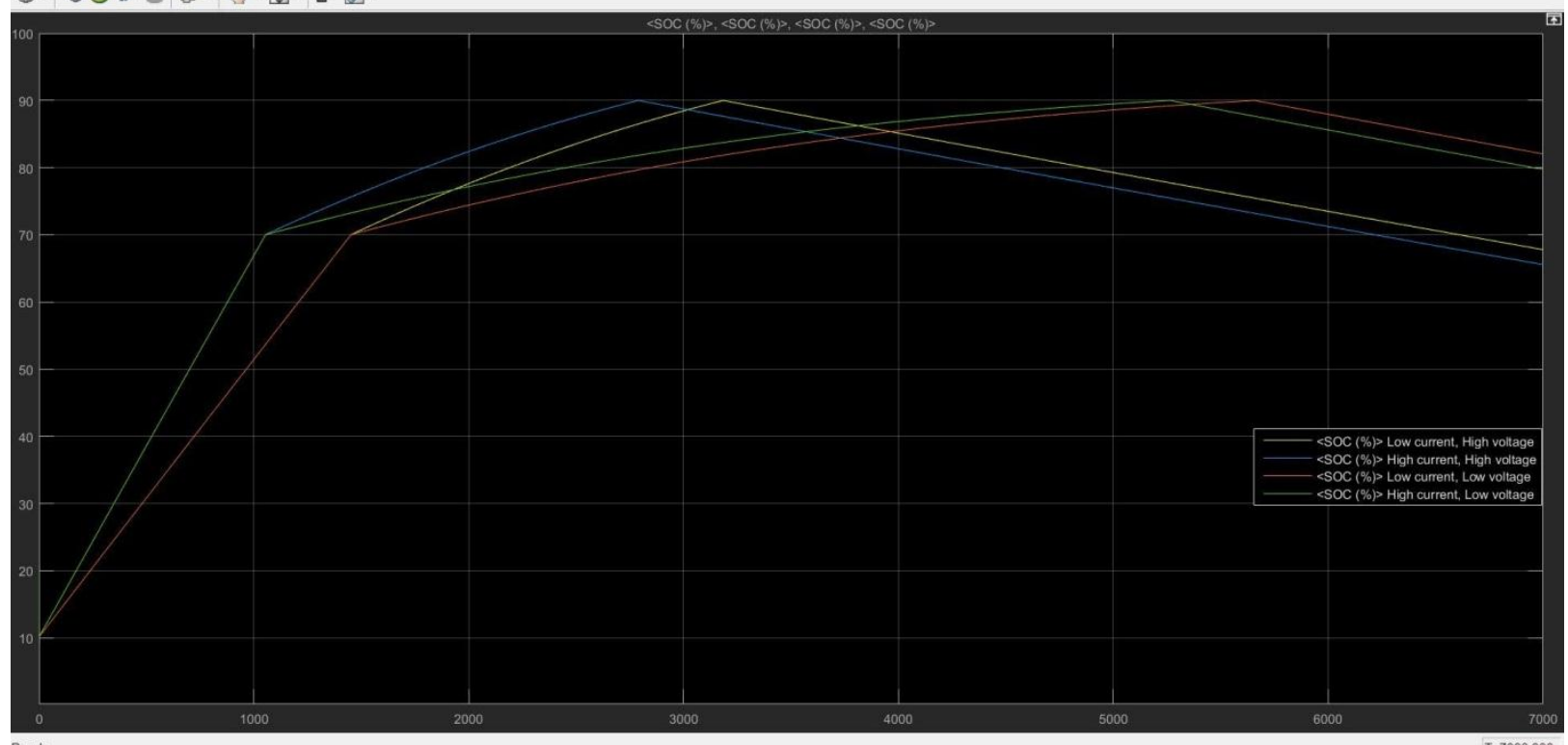

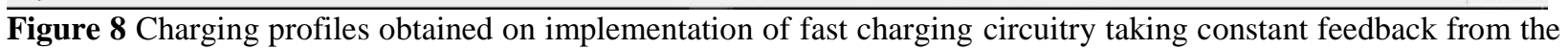
battery 


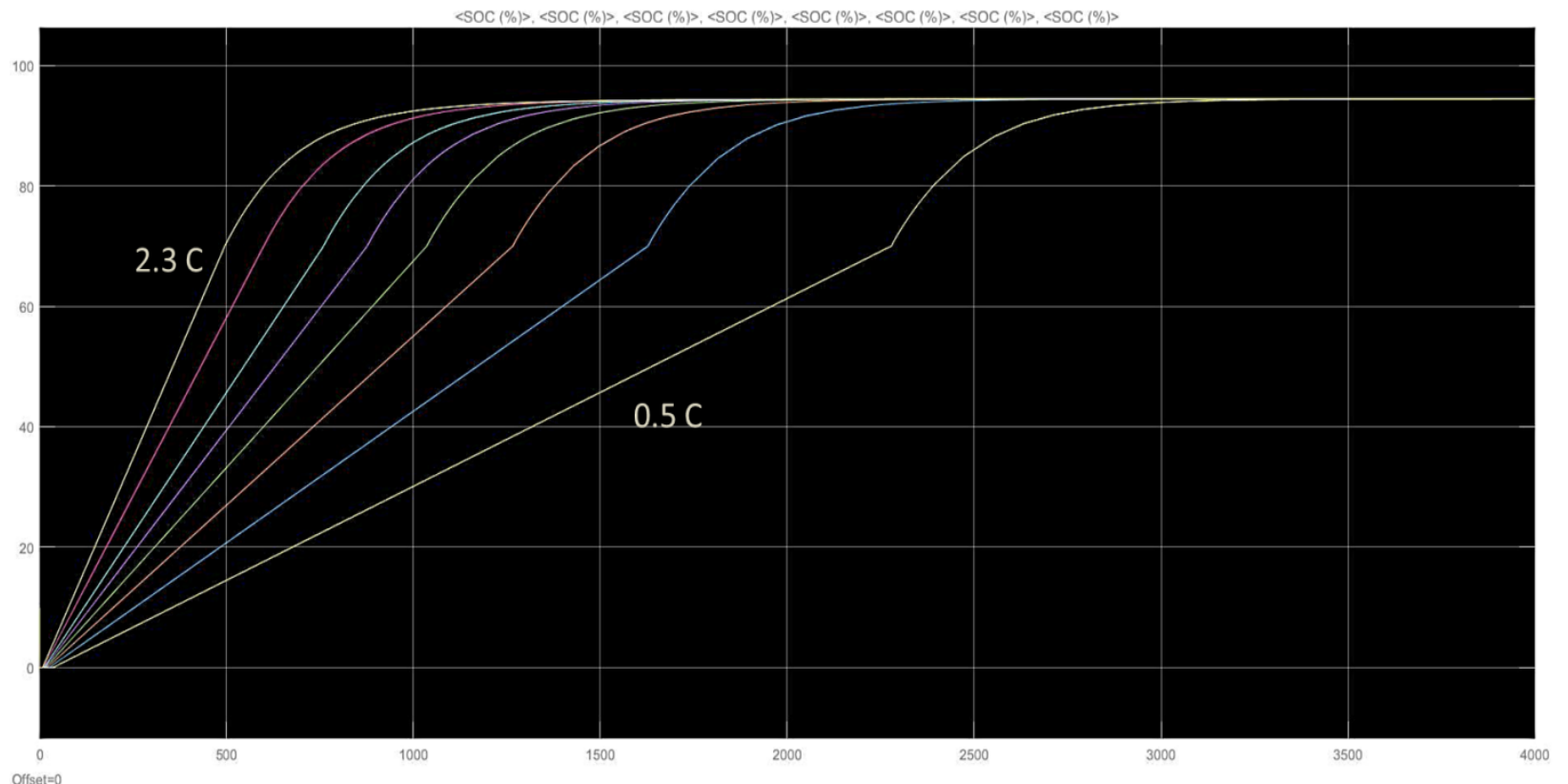

Figure 9 Charging characteristics with varying C-rate

\section{Results and further scope of improvement}

As a result of the experiments and the simulations conducted, the direct inference result is that varying the constant current mode C-rate for charging has direct effect on both charging time and battery degradation. The charging characteristics with varying C-rates are displayed in Figure 9 above. Fast charging can reduce charging time by up to only 15 minutes for 20 to $90 \%$ but the degradation is large. The charger can be optimized to fast charge, but only to a limited extent, limited by the battery degradation. An effective negotiation is charging at $1.1 \mathrm{C}$ to $1.5 \mathrm{C}$ during constant current mode, yielding a charging time of about 25-35 minutes for 20 to $90 \%$ charging with much less degradation.

However, there is still quite a lot of scope of improvement ranging from improvement in charging time by charging at different constant current for different SOC of the battery. This includes charging at higher C-rates for lower values of SOC and gradually decreasing the C-rate as SOC increases.

Moreover, a well-developed mathematical battery degradation model can be taken into consideration for improvement of the accuracy of the results. Circuit non-idealities also contribute to the inaccuracies in theoretical analysis of the procedure.

\section{Acknowledgment None.}

\section{Conflicts of interest}

The authors have no conflicts of interest to declare.

\section{References}

[1] Gao L, Liu S, Dougal RA. Dynamic lithium-ion battery model for system simulation. IEEE Transactions on Components and Packaging Technologies. 2002; 25(3):495-505.

[2] Liaw BY, Nagasubramanian G, Jungst RG, Doughty DH. Modeling of lithium ion cells-a simple equivalent-circuit model approach. Solid State Ionics. 2004; 175(1):835-9.

[3] Bhuvaneswari K, Niruba K. A single-phase ac-dc converter with improved efficiency using atmega8 microcontroller. International Journal of Innovative Research in Science, Engineering and Technology. 2014; 3(5): 12537-42.

[4] Ramesh R, Subathra U, Ananthi M. Single phase ACDC power factor corrected converter with high frequency isolation using buck converter. 2014; 4(3): 79-82.

[5] Dearborn S. Charging Li-ion batteries for maximum run times. Power Electronics Technology. 2005; 31(4):40-9.

[6] Ansean González D, García Fernández VM, González Vega M, Viera Pérez JC, Álvarez Antón JC, Blanco Viejo CJ. Efficient fast-charging strategies for $\mathrm{Li}$-ion batteries. In28th international electric vehicle symposium and exhibition 2015. 
[7] Wu X, Maeda T, Fujimoto H, Ishii S, Fujita K. Threephase high frequency transformer isolated $\mathrm{AC}$ to $\mathrm{DC}$ converter for EV battery quick charging. In international power electronics and motion control conference 2012 (pp. 643-7). IEEE.

[8] Wang J, Li Q, Song P, Hou P. Analysis of output characteristics of buck converter. Energy and Power Engineering. 2013; 5(4):1069.

[9] Chen LR, Liu CS, Chen JJ. Improving phase-locked battery charger speed by using resistance-compensated technique. IEEE Transactions on Industrial Electronics. 2009; 56(4):1205-11.

[10] Chen M, Rincón-Mora GA. Accurate, compact, and power-efficient Li-ion battery charger circuit. IEEE Transactions on Circuits and Systems II: Express Briefs. 2006; 53(11):1180-4.

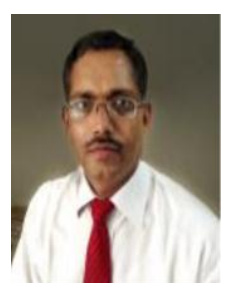

Prof. Dheerendra Singh hails from Uttar Pradesh. He graduated (B.E. Electrical Engineering) from IIT Roorkee (Formerly University of Roorkee), Post graduated from IIT Delhi \& obtained Doctorate from BITS-Pilani. He is having more than 20 yrs of teaching and Industrial experience. He joined BITS in 1999 as a faculty of EEEDepartment. He is more involved in teaching and research in the area of Power Electronics based Renewable Energy Systems, Controls \& Drives. He successfully co-ordinated many graduate programme for the industries like NTPC, $\mathrm{CFCl}$, Hindustan Zinc etc.

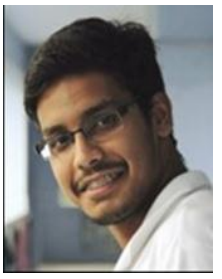

Shivam Khandelwal is an undergraduate third year student at BITS, Pilani, Pilani campus, born in Alwar (Raj.). He is an active member of IEEE BITS Pilani chapter and is interested in battery research, electric and hybrid vehicles, and data science, particularly big data analytics. He has authored two other publications, entitled 'Battery selection for plug-in hybrid electric vehicles', presented at International Conference on Advanced Research Applications in Engineering and Technology held during June 2015 and "Constant Current Controlled Feedback Based Lithium - Ion Battery Charger" at National Seminar on "Best Practices for Power Generation, Transmission and Distribution (NSBPPGTD)" during January 2016. He aims to develop an indigenously developed electric vehicles run ecosystem in India.

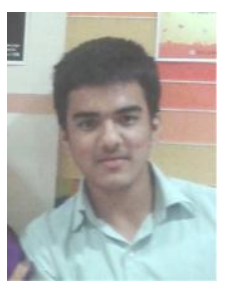

Parakh Sarda is an undergraduate third year student of Electrical and Electronics department at BITS Pilani, Pilani campus. He was born at Raipur (C.G.) and is interested in fields of architecture for processors, digital logics and embedded designing. He is the author of one paper titled "Constant

Current Controlled Feedback Based Lithium - Ion Battery Charger" presented at National Seminar on "Best Practices for Power Generation, Transmission and Distribution (NSBPPGTD)" during January 2016. He was selected as Summer Research Fellow[2016] from the Indian National Science Academy and is currently in IIT Madras working on Instruction Pre-fetching based on Branch Prediction Algorithms on BlueSpec software under Dr.V.Kamakoti. He aims to pursue higher study in the field of VLSI architecture. 